\title{
COVID-19: implicações e aplicações da Psicologia Positiva em tempos de pandemia
}

\author{
COVID-19: implications and applications of \\ Positive Psychology in times of pandemic
}

\author{
Cristian ZANON ${ }^{1}$ (iD) 0000-0003-3822-5275 \\ Letícia Lovato DELLAZZANA-ZANON² (iD) 0000-0003-0649-1675 \\ Solange Muglia WECHSLER ${ }^{2}$ (D) 0000-0002-9757-9113 \\ Rodrigo Rodrigues FABRETTI' (iD) 0000-0002-8342-871X \\ Karina Nalevaiko da ROCHA ${ }^{2}$ (iD) 0000-0002-8324-7847
}

\section{Resumo}

A pandemia causada pela dispersão da COVID-19 no mundo obrigou muitos países a adotarem o isolamento social como medida de contenção do vírus. Pesquisas prévias indicam que pessoas submetidas ao isolamento social desenvolvem sintomas psicológicos variados, principalmente relacionados ao estresse, ansiedade e depressão, decorrentes da privação social e do confinamento. Este artigo objetiva apresentar pesquisas produzidas pela Psicologia Positiva e indicar como esses achados podem subsidiar intervenções para a promoção de saúde mental e bem-estar durante o isolamento social. Dentre os construtos descritos, destacam-se pesquisas sobre autocompaixão, resiliência, criatividade, otimismo, esperança, bem-estar subjetivo e práticas de meditação mindfulness para lidar com os efeitos adversos do isolamento. Considerações e implicações dessas práticas são discutidas em detalhes.

Palavras-chave: Atenção plena; Criatividade; Otimismo; Intervenções; Isolamento social.

\footnotetext{
$\boldsymbol{\nabla} \mathbf{\nabla} \boldsymbol{\nabla}$

${ }^{1}$ Universidade Federal do Rio Grande do Sul, Departamento de Psicologia do Desenvolvimento e da Personalidade, Programa de Pós-Graduação em Psicologia. R. Ramiro Barcelos, 2600, Santa Cecília, 90035-003, Porto Alegre, RS, Brasil. Correspondência para/ Correspondence to: C. ZANON. E-mail: <crstn.zan@gmail.com>.

2 Pontifícia Universidade Católica de Campinas (PUC-Campinas), Centro de Ciências da Vida, Programa de Pós-Graduação em Psicologia. Campinas, SP, Brasil.

$\mathbf{v} \mathbf{v}$

Como citar este artigo/How to cite this article

Zanon, C., Dellazzana-Zanon, L. L., Wechsler, S. M., Fabretti, R. R., \& Rocha, K. N. (2020). COVID-19: implicações e aplicações da Psicologia Positiva em tempos de pandemia. Estudos de Psicologia (Campinas), 37, e200072. https://doi.org/10.1590/19820275202037 e200072
} 


\begin{abstract}
The pandemic caused by the dispersion of COVID-19 in the world has forced many countries to adopt quarantines to contain the virus. Previous researches indicate that people subjected to social isolation develop psychological symptoms mainly related to stress, anxiety, and depression due to social deprivation and confinement. This manuscript presents researches produced by Positive Psychology and indicates how these findings can support interventions to promote mental health and well-being during quarantines. We highlight researches focused on self-compassion, resilience, creativity, hope, optimism, subjective well-being, and mindfulness meditation practices to deal with the adverse effects of quarantines. Considerations and implications of these practices are discussed in detail.
\end{abstract}

Keywords: Mindfulness; Creativity; Optimism; Interventions; Social isolation.

No final de dezembro de 2019, foi identificado um novo tipo de pneumonia - Coronavirus Disease 2019 (COVID-19) -, causada pelo vírus Severe Acute Respiratory Syndrome Coronavirus 2 (SARS-CoV-2, Coronavírus da Síndrome Respiratória Aguda Grave 2) (Huang et al., 2020; Li et al., 2020; Wang et al., 2020b; Wu et al., 2020). Embora a severidade da doença não esteja totalmente clara, estimativas sugerem que a probabilidade de morte após o desenvolvimento dos sintomas da COVID-19 é de 1,4\% (Wu et al., 2020). Devido à rápida propagação da doença, vários países adotaram o isolamento social como medida para reduzir a dispersão do vírus (World Health Organization [WHO], 2020). Essa medida envolve mudanças na rotina e perda de liberdade. Evidências recentes indicam impactos psicológicos devido à determinação do isolamento social como medida de contenção a epidemias (Desclaux, Badji, Ndione, \& Sow, 2017; Jeong et al., 2016, Wang et al., 2020a). Sintomas psicopatológicos de estresse pós-traumático, ansiedade e depressão podem acometer, principalmente, profissionais de saúde e pessoas de baixa renda (Brooks et al., 2020). Devido à possibilidade desse cenário ser estendido à população geral na pandemia de COVID-19, faz-se necessário o desenvolvimento de intervenções eficientes para reduzir o sofrimento psicológico das pessoas. Para contribuir com tal demanda, este artigo objetiva apresentar pesquisas psicológicas, principalmente provindas da Psicologia Positiva, que possam orientar no enfrentamento dos efeitos adversos (e.g., sintomas psicopatológicos e redução do bem-estar) produzidos pelo isolamento social.

\title{
Psicologia Positiva
}

A Psicologia Positiva caracteriza-se como uma área da ciência psicológica que investiga: (a) a experiência subjetiva positiva, (b) traços individuais positivos e (c) instituições positivas (Ivtzan, Lomas, Hefferon, \& Worth, 2016; Lopez \& Snyder, 2009; Seligman \& Csikszentmihalyi, 2000). De acordo com esses autores, o foco em aspectos positivos de pessoas e instituições visa melhorar a qualidade de vida e prevenir psicopatologias provindas de limitações e de uma vida sem sentido. Diferente da tradicional abordagem psicológica focada em reduzir o sofrimento e adequar o comportamento desviante, a Psicologia Positiva busca promover o desenvolvimento das virtudes e potencialidades humanas (Peterson \& Seligman, 2004) por meio de intervenções baseadas em evidências (Smirnova \& Parks, 2018).

Devido aos resultados promissores das intervenções positivas em contextos clínicos e não clínicos, desenvolveu-se a Psicoterapia Positiva (Rashid \& Seligman, 2018), constituindo-se como uma terapia psicológica que combina a abordagem e técnicas da Terapia Cognitivo-Comportamental (Beck, 2011) com achados das intervenções positivas. A Psicoterapia Positiva enfatiza o desenvolvimento de forças e virtudes nos pacientes, como forma de auxiliá-los a lidar com situações-problema, reduzir seu sofrimento e desenvolver uma vida mais satisfatória e com mais sentido (Peterson \& Seligman, 2004; Rashid \& Seligman, 2018). Para fins históricos, ressalta-se que os psicólogos humanistas já enfatizavam aspectos positivos do indivíduo na clínica. 


\section{Considerações e esclarecimentos sobre a proposta}

Duas grandes áreas de investigação da Psicologia Positiva são o bem-estar e as forças e virtudes humanas (Hutz, Midgett, Pacico, Bastianello, \& Zanon, 2014; Ivtzan et al., 2016; Seligman \& Csikszentmihalyi, 2000; Smirnova \& Parks, 2018; Zanon, 2017). Com o intuito de indicar como os achados da Psicologia Positiva podem contribuir para a situação de isolamento social, confinamento e perda da liberdade produzidos pela dispersão da COVID-19 no mundo, este artigo apresenta alguns construtos psicológicos e a maneira como eles podem ser desenvolvidos em diferentes contextos, seja por psicoterapia presencial ou via Internet, seja de forma independente. São abordados neste trabalho os seguintes construtos: resiliência (i.e., capacidade de lidar com adversidades), autocompaixão (i.e., cuidado e empatia consigo mesmo), criatividade (i.e., desenvolvimento do potencial humano para gerar ideias novas e relevantes), otimismo (i.e., expectativa positiva de mudanças futuras), esperança (i.e., identificação de metas relevantes, desenvolvimento de estratégias e persistência para alcançar as metas), bem-estar subjetivo (i.e., felicidade) e mindfulness (i.e., meditação de atenção plena).

O presente artigo considera importante que a implementação dessas práticas seja acompanhada por psicólogos, principalmente em casos de diagnósticos psiquiátricos ou de grande sofrimento psicológico, pela possibilidade do clínico poder tratar outras questões relevantes urgentes. A seguir, serão apresentados estudos sobre grupos de risco e fatores que podem produzir efeitos psicológicos adversos decorrentes do isolamento social. Embora alguns desses estudos não relatem efeitos causados pela dispersão da COVID-19 e tenham sido desenvolvidos em outros contextos, acredita-se que eles sejam úteis para contextualizar os efeitos psicológicos sofridos em tempos da pandemia de COVID-19.

\section{Efeitos psicológicos do isolamento social}

Em uma recente revisão da literatura sobre os impactos psicológicos do isolamento social em participantes de dez países que foram submetidos ao isolamento por terem sido expostos ao Ebola, Influenza H1N1, Severe Acute Respiratory Syndrome (SARS, Síndrome Respiratória Aguda Grave) e Middle East Respiratory Syndrome (MERS, Síndrome Respiratória do Oriente Médio), dentre outros vírus de epidemias/pandemias anteriores, foram identificados alguns fatores preditivos de sofrimento psicológico (Brooks et al., 2020). Fatores como apresentar histórico de transtorno psiquiátrico prévio e ser profissional de saúde, por exemplo, predisseram emoções negativas duradouras. Os profissionais de saúde, além de apresentarem uma diversidade de emoções negativas após o isolamento social (i.e., tristeza, nervosismo, culpa), relataram sofrer maior estigmatização do que outros profissionais (Hawryluck et al., 2004). Esses achados sugerem maior propensão a sofrimento mental nesse grupo e, consequentemente, maior necessidade de intervenção.

Outros fatores identificados como preditores de maior sofrimento mental foram a duração do período de isolamento social (> 10 dias), medo de ser infectado ou infectar outras pessoas, frustração, tédio, falta de suprimentos e informações inadequadas sobre a doença (Brooks et al., 2020). Alguns desses fatores foram associados a um maior número de sintomas de Transtorno de Estresse Pós-Traumático (TEPT), transtornos de ansiedade e afetos negativos após o final do isolamento social (Desclaux et al., 2017; Hawryluck et al., 2004; Jeong et al., 2016; Reynolds et al., 2008).

Outras condições adversas decorrentes do isolamento social, como perdas financeiras e estigma, foram identificados como fatores de risco para o desenvolvimento de transtornos mentais e afetos negativos prolongados (Bai et al., 2004; Mihashi et al., 2009). Os prejuízos financeiros, por sua vez, parecem constituir maior fator de risco no desenvolvimento de transtornos mentais para famílias de nível socioeconômico baixo (Hawryluck et al., 2004). Isso pode decorrer do maior impacto que a perda de renda representa para essas famílias (Brooks et al., 2020). 
Evidências indicam que $30 \%$ da amostra de alguns estudos sobre participantes submetidos ao isolamento social apresentavam critérios para o diagnóstico de TEPT (Hawryluck et al., 2004; Sprang \& Silman, 2013). Cabe ressaltar que essa prevalência é similar à encontrada em sobreviventes de guerra (Morina, Stam, Pollet, \& Priebe, 2018). Tais achados reforçam a necessidade de medidas concretas para dirimir os impactos psicológicos da pandemia. Uma possível explicação para a grande prevalência de sintomas de trauma em indivíduos expostos a pandemias e que enfrentaram períodos de isolamento social pode ser decorrente do medo e da percepção de risco, que podem ser amplificados por informações pouco claras - comuns nos períodos iniciais de surtos (Johal, 2009). Outros fatores relevantes podem ser a ausência de vacinas e remédios, bem como o desconhecimento da doença.

A cobertura de pandemias por fontes de notícias autênticas constitui um fator desencadeante de medo, conforme observado em um estudo sobre a gripe aviária de 2005 (van den Bulck \& Custers, 2009), pois indica que algo importante e, talvez, difícil de controlar possa estar ameaçando a população. Nesse estudo, constatou-se que maior exposição à televisão foi associada a maior medo da doença.

Dados atuais revelaram que sintomas físicos como febre persistente, calafrios e dor de cabeça, dentre outros, se associaram a um maior impacto psicológico da atual pandemia de COVID-19 (Wang et al., 2020a). Os autores também identificaram que a percepção negativa sobre status de saúde e histórico de doença crônica também se associaram a níveis significativamente maiores de sintomas de TEPT, estresse, ansiedade e depressão, ao passo que o uso de máscaras, como medida protetiva, associou-se a menores níveis de ansiedade e depressão. Nas próximas seções, serão apresentados construtos estudados pela Psicologia Positiva e indicações de sua importância para lidar com o contexto de isolamento social.

\section{Resiliência}

Ao contrário do que se pode pensar, a Psicologia Positiva também se dedica a pesquisar algumas das experiências humanas mais difíceis e dolorosas (Ivtzan et al., 2016). Por exemplo, todos sofrem perdas de pessoas queridas ao longo da vida. Entretanto, enquanto algumas pessoas experimentam um sofrimento profundo e têm muita dificuldade para se recuperar, outras conseguem suportar bem as perdas ou os eventos potencialmente traumáticos, sem interrupção aparente de sua capacidade de trabalhar ou de manter relacionamentos próximos (Bonanno, 2004).

Situações adversas podem fornecer a possibilidade do indivíduo aprender com seus esforços para tornar-se psicologicamente mais forte e crescer como ser humano (Ivtzan et al., 2016). Uma das formas de tentar entender como os indivíduos podem enfrentar de forma positiva as situações adversas da vida é por meio do estudo da resiliência. A resiliência refere-se a uma classe de fenômenos caracterizados por padrões de adaptação positiva no contexto de adversidades ou riscos significativos para o desenvolvimento (Luthar, Lyman, \& Crossman, 2014; Masten, 2001; Masten \& Reed, 2002). Recentemente, a resiliência tem sido considerada como o fenômeno pelo qual muitas pessoas mantêm sua saúde mental, apesar da exposição a adversidades psicológicas ou físicas (Kalisch et al., 2017). Em ambas as definições, a exposição a riscos ou adversidades graves é um pré-requisito central da resiliência (Luthar, Cicchetti, \& Becker, 2000; Masten, 2001). A pandemia de COVID-19 constitui uma crise grave que tem afetado toda a população mundial de diferentes formas (e.g., perda de familiares, demissões, restrição financeira, isolamento social e perda da liberdade). Nesse sentido, pode-se pensar que se está vivendo um momento ímpar no qual a resiliência pode constituir um processo essencial para o enfrentamento das adversidades derivadas da pandemia. A incorporação de ações no cotidiano que envolvam autocompaixão, criatividade, otimismo e meditação pode promover resiliência e bem-estar em muitas pessoas. As próximas seções abarcarão esses temas. 


\section{Autocompaixão}

A autocompaixão envolve direcionar a si próprio o mesmo tipo de cuidado, bondade e compaixão transmitidos às pessoas queridas que estão sofrendo (Neff, 2003b). De acordo com a autora, a autocompaixão está relacionada a uma atitude emocionalmente positiva direcionada para si mesmo, que pode proteger contra as consequências negativas do autojulgamento, do isolamento social e da ruminação. A autocompaixão é composta por três elementos: bondade consigo mesmo (versus autocrítica severa), senso de humanidade (versus isolamento social) e atenção plena (versus superidentificação; Neff, 2003a, 2003b). Segundo Neff (2003b), a bondade consigo refere-se a ser gentil e compreensivo consigo mesmo em situações de sofrimento ou fracasso, em vez de ser severamente autocrítico; o senso de humanidade diz respeito a perceber as próprias experiências como parte da experiência humana mais ampla, em vez de vê-las como separadoras e isoladoras; a atenção plena, por sua vez, está relacionada a manter pensamentos e sentimentos dolorosos em consciência equilibrada, ao invés de se identificar demais com eles.

A autocompaixão, de fato, parece associada a resultados positivos em diferentes domínios, como afetos, padrões cognitivos, realizações e conexões sociais (Barnard \& Curry, 2011). No contexto brasileiro, verificou-se que a autocompaixão apresenta relações positivas e moderadas com a autoestima e a autoeficácia (Souza \& Hutz, 2016) - o que indica que pessoas com escores mais elevados de autocompaixão também apresentam maior senso de autovalor e crença em suas capacidades de executar tarefas. Em relação a psicopatologias, evidências indicam que a autocompaixão amortece o impacto de eventos negativos, pois as pessoas autocompassivas parecem perceber os eventos negativos de maneira a reduzir seu impacto (Leary, Tate, Adams, Allen, \& Hancock, 2007), além de apresentarem menores níveis de depressão, ansiedade e estresse (Souza, Policarpo, \& Hutz, 2020).

A autocompaixão parece bastante relevante para o enfrentamento de situações dolorosas que estão fora do controle do indivíduo (Neff \& Germer, 2017), uma vez que as pessoas autocompassivas têm menor probabilidade de catastrofizar situações negativas, sentir ansiedade após um estressor e evitar tarefas desafiadoras por medo do fracasso (Allen \& Leary, 2010). Com autocompaixão, por meio do exercício da bondade consigo, do senso de humanidade e da atenção plena, é possível assumir uma posição de cuidado em relação a si mesmo, essencial para enfrentar períodos de crise.

No contexto da pandemia causada pela dispersão da COVID-19, parece mais aceitável pensar que se está isolado, sem contato com outras pessoas, quando se considera que outros também estão nesse contexto. Assim, compreender que outras pessoas também estão sofrendo consequências adversas em função da COVID-19, como perda de emprego ou de seus familiares, pode contribuir para um sentimento de conexão e perspectiva da própria situação. Ou seja, lembrar a natureza compartilhada do sofrimento diminui a sensação de isolamento e sinaliza que as coisas poderiam ser piores (Neff \& Germer, 2017).

\section{Criatividade, otimismo e esperança}

Frente às situações inesperadas e adversas, como o aparecimento de uma pandemia, surge a busca de soluções e alternativas ainda não testadas. A criatividade, nessas situações, torna-se essencial para vencer os momentos de crise, tornando-se uma força de saúde mental (Gillam, 2013; Glaveanu, 2014; Oliveira, Nakano, $\&$ Wechsler, 2016), na medida em que traz consigo uma atitude de otimismo e esperança de dias melhores.

A compreensão da criatividade evoluiu, com o passar do tempo, indicando a sua importância não somente para a área artística (González-Rey, 2018). Estratégias criativas podem ser desenvolvidas com pessoas de diferentes faixas etárias, visando desenvolver o potencial criativo com objetivos pessoais (Almeida \& Wechsler, 2015; Mundim, Wechsler, \& Morais, 2020), educacionais (Fleith, 2019; Morais \& Almeida, 2019) e 
profissionais (Campos, 2016; Gondim et al., 2015; Nakano \& Wechsler, 2018). Dessa maneira, caraterísticas como abertura às novas experiências, persistência, tolerância às ambiguidades, inconformismo, uso da intuição e imaginação, geralmente, estão presentes em uma pessoa criativa (Kaufman \& Sternberg, 2010; Runco \& Pritzker, 2020). Tais características são fundamentais para ajudar no enfrentamento de situações de crise, pois estas passam a ser percebidas como oportunidades para crescimento, ao invés de momentos de desalento (Nakano, Machado, \& Abreu, 2019).

Na visão da Psicologia Positiva, a criatividade é um fenômeno legítimo e passível de investigação psicológica, que se agrega a outros fenômenos como a felicidade, a autorrealização e as emoções e sentimentos positivos, dentre outros (Csikszentmihalyi, 2014). Assim sendo, a criatividade é considerada uma das forças de caráter que permitem ao indivíduo um funcionamento ideal, firmando-se como um fator de proteção (Peterson, Ruch, Beermann, Park, \& Seligman, 2007; Snyder \& Lopez, 2008). É importante ressaltar que todos possuem potencial criativo, nas mais diferentes áreas, e que este pode ser desenvolvido, principalmente quando existe um ambiente estimulador (Wechsler, 2008). Nesse sentido, faz-se relevante descobrir a criatividade que possui cada indivíduo e as pessoas que o cercam. Isso pode resultar na criação e implementação de estratégias, em nível pessoal e interpessoal, que possibilitem conduzir ao desenvolvimento das próprias habilidades criativas pessoais bem como daquelas das pessoas ao redor, como crianças, adolescentes e idosos. Por sua vez, a criatividade pode auxiliar na busca de alternativas para colaborar com a solução dos problemas sociais gerados pela pandemia.

O otimismo é uma das características presentes em pessoas criativas, pois crises e problemas são vistos como desafios e oportunidades para novas aprendizagens. O otimismo pode ser definido como uma força capaz de manter a persistência e resistência do indivíduo (Peterson \& Steen, 2002). Níveis elevados de otimismo têm sido relacionados ao bem-estar subjetivo (Gallagher, Lopes, \& Pressman, 2013). Como o otimismo se caracteriza por expectativas positivas, outros construtos, como a autoeficácia e a esperança, também podem estar relacionados a ele, sendo considerados construtos complementares (Robinson \& Snipes, 2009).

A relação entre otimismo e criatividade vem sendo pesquisada há várias décadas, desde os trabalhos de Osborn (1963), com técnicas como tempestade de ideias para gerar novas soluções para um problema, ou pelas estratégias conhecidas como Resolução Criativa de Problemas (Parnes, 1967), que visam enfocar um problema de forma flexível, ou seja, sob diferentes pontos de vista. Vários estudos indicam a relação positiva entre otimismo, esperança, criatividade e autoconfiança para o bem-estar de um indivíduo, tanto em nível pessoal quanto profissional (Snyder \& Lopez, 2008). Considerando que ter uma expectativa positiva do futuro e usar a imaginação são essenciais para a criatividade (Santos, Gibim, \& Wechsler, 2020), recomendase que essas atitudes sejam desenvolvidas frente aos desafios de uma pandemia para o estabelecimento do equilíbrio e da saúde mental. Estratégias que utilizam a visualização, por meio das quais o indivíduo possa se situar em um futuro, por exemplo, daqui a cinco ou até dez anos, permitem perceber o momento atual como uma aprendizagem de vida na qual foi preciso mudar os hábitos e padrões de relacionamento.

\section{Bem-estar subjetivo}

O Bem-Estar Subjetivo (BES) é composto por um fator cognitivo (e.g., satisfação de vida) e dois fatores emocionais (e.g., afetos positivos e afetos negativos) que definem o nível de felicidade percebida (Diener et al., 2016). A satisfação de vida caracteriza-se por uma avaliação cognitiva do nível de contentamento com amplos aspectos relevantes da vida, como, por exemplo, família, trabalho e relações sociais. Afetos positivos são compostos pela frequência e intensidade com que alguém vivencia emoções positivas, como entusiasmo e alegria; já afetos negativos são definidos pela frequência e intensidade com que alguém vivencia emoções como angústia, tristeza e medo (Watson \& Clark, 1994). Nessa perspectiva, uma pessoa com alto nível de 
bem-estar subjetivo (ou feliz) é aquela que apresenta altos níveis de satisfação com a vida, alta frequência de afetos positivos e baixa frequência de afetos negativos. Por sua vez, uma pessoa com baixos níveis de BES (ou infeliz) apresenta baixa satisfação com a vida, baixa frequência de afetos positivos e alta frequência de afetos negativos.

Até meados dos anos 80 e 90 do século XX, as pesquisas sobre felicidade eram consideradas de pouco valor, sem importância ou mesmo irrelevantes (Lyubomirsky, 2019) por não se considerar possível aumentar os níveis de felicidades das pessoas (devido à adaptação hedônica) e, também, por não se encontrarem relações relevantes entre felicidade e variáveis suspostamente importantes (como renda, status social, dentre outras; Lyubomirsky, 2007). A autora menciona que a nova estratégia de pesquisa foi, então, selecionar pessoas altamente felizes e avaliar o que elas apresentavam em comum. O resultado foi de que estas pessoas eram mais sociáveis, gratas, empáticas e que usavam seu tempo para ajudar os outros (Lyubomirsky \& Layous, 2013). Pesquisas descritivas e correlacionais suportaram esses achados em estudos subsequentes (Lyubomirsky, 2007), mas eram inconclusivas sobre a direção causal da relação: a felicidade produzia comportamentos sociais e altruístas ou eram esses comportamentos que produziam aumento da felicidade? Novas evidências provindas de estudos experimentais em diferentes amostras, no entanto, indicam que as pessoas podem aumentar seus níveis de felicidade por meio de comportamentos intencionais que envolvem gratidão, empatia e altruísmo e, também, que conseguem mantê-los, em muitos casos, por um longo período de tempo (Sheldon \& Lyubomirsky, 2019).

Além de ser algo muito desejado por muitas pessoas (Diener, 2000), apresentar altos níveis de BES está associado com maior longevidade, melhores condições de saúde (Diener \& Chan, 2011) e menores níveis de psicopatologias (Pelechano, González-Leandro, Garcia, \& Morán, 2013). Duas investigações, conduzidas no Brasil, também suportam esses achados. Um estudo correlacional verificou que pessoas mais satisfeitas com suas vidas apresentam menores níveis de depressão, ansiedade e estresse (Zanon, 2017). Outro estudo longitudinal, conduzido antes e depois do incêndio da boate Kiss em Santa Maria (RS), em 2013, verificou que universitários mais felizes apresentaram menor vulnerabilidade à ruminação, ansiedade e estresse póstraumático cinco meses após o incêndio (Zanon, Hutz, Reppold, \& Zenger, 2016). Esses achados suportam a hipótese de que o aumento do BES pode contribuir para manter a saúde mental e reduzir sintomas psicopatológicos durante e após a pandemia de COVID-19. Assim sendo, práticas que procurem encontrar pontos positivos na experiência de cada dia podem ser benéficas para o aumento do BES.

Diferentemente do que se acreditava, a felicidade é pouco influenciada pela renda, status social e aparência física, mas é bastante influenciada pela genética, por fatores intencionais relacionados à ação e pela personalidade (Lyubomirsky, Sheldon, \& Schkade, 2005). Evidências verificaram que pessoas mais extrovertidas tendem a ser mais felizes por experienciarem mais afetos positivos que pessoas mais tímidas, bem como pessoas com altos níveis de neuroticismo tendem a ser menos felizes por experienciarem mais afetos negativos que pessoas mais emocionalmente estáveis (Hutz et al., 2014; Rosin, Zanon, \& Teixeira, 2014). Ressalta-se, entretanto, que as pessoas mais introvertidas ou com níveis mais elevados de neuroticismo (ou com ambos) podem beneficiar-se das práticas de autocompaixão, otimismo e meditação mindfulness, dentre outras, para aumentar o BES. Contudo, é possível que seja necessário maior persistência e dedicação dessas pessoas às práticas para obtenção dos resultados esperados, se comparadas às pessoas mais extrovertidas e com baixos níveis de neuroticismo.

\section{Mindfulness, psicopatologia e bem-estar}

A meditação mindfulness, ou de atenção plena, é originalmente uma prática espiritual budista que tem sido amplamente implementada de forma secular em intervenções voltadas para o aumento do bem-estar 
(Good et al., 2016). Mindfulness é, comumente, conceituada como uma tomada de consciência que surge de um direcionamento intencional da atenção a experiências presentes sem julgá-las (Bishop et al., 2004). Mindfulness estrutura-se em três componentes: intenção, atenção e atitude. Intenção reflete o objetivo que o participante deseja com a prática (e.g., autorregulação emocional, autorreflexão). Atenção reflete o estado de tornar-se, totalmente, consciente da experiência interna momento após momento. Atitude descreve a qualidade da atenção do praticante, podendo ser repleta de aceitação, ternura e curiosidade (Ivtzan et al., 2016).

Dois estudos recentes demonstram a importância da meditação no contexto da sintomatologia depressiva. Em uma meta-análise de 25 ensaios clínicos randomizados sobre intervenções baseadas em mindfulness, os autores verificaram efeitos moderados para a prevenção de sintomas depressivos em universitários (Ma, Zhang, \& Cui, 2019). Em uma intervenção desenvolvida no contexto brasileiro para redução de depressão e ansiedade ao longo de seis semanas, verificou-se a atenuação de sintomas depressivos e ansiogênicos após seis meses (para meditadores ativos), sendo que o efeito de redução da ansiedade propagou-se mesmo depois de um ano da intervenção nesse grupo (Carpena, Tavares, \& Menezes, 2019).

Além de reduzir os sintomas psicopatológicos, a meditação parece produzir efeitos importantes no bem-estar das pessoas porque contribui para que o praticante mude e controle a autopercepção do mundo e eventos ao seu redor, ao invés de tentar mudar o mundo e as experiências em si (Menezes, Dell'Aglio, \& Bizarro, 2011). Uma revisão sistemática recente da relação entre mindfulness e saúde psicológica (Tomlinson, Yousaf, Vittersø, \& Jones, 2018) indica associação positiva entre esses construtos em 13 estudos avaliados. Ademais, evidências da relação entre mindfulness e bem-estar psicológico foi demonstrada em várias amostras (Bajaj, Gupta, \& Pande, 2016; Bergin \& Pakenham, 2016; Christie, Atkins, \& Donald, 2017; Zimmaro et al., 2016).

A prática de meditação mindfulness, comportamentos de empatia, generosidade e gratidão para com os outros podem representar importantes atividades para o aumento do BES durante a pandemia. As práticas de meditação foram apresentadas neste artigo pelo fato de, no contexto de uma pandemia, serem facilmente implementadas em casa. Já os outros comportamentos, por sua vez, podem ocorrer como parte de processos psicoterapêuticos, seja com acompanhamento psicológico, seja de forma autônoma. Ressaltase que demonstrar empatia, generosidade e altruísmo, juntamente com o desenvolvimento de otimismo, criatividade, esperança, autocompaixão e resiliência, também pode contribuir substancialmente para o aumento do BES (Zanon, Dellazzana-Zanon, \& Hutz, 2014).

\section{Discussão}

Este artigo objetivou apresentar achados da Psicologia Positiva que podem contribuir para a redução de sintomas psicopatológicos (e.g., depressão, ansiedade e estresse) e o aumento do bem-estar durante o período de isolamento social causado pela dispersão da COVID-19. Esses achados podem subsidiar intervenções nos mais diversos contextos, a partir da combinação de estratégias para promover autocompaixão, criatividade, otimismo e bem-estar (por meio de práticas de meditação mindfulness), por exemplo. Tais estratégias podem também ocorrer no contexto clínico, mediante o desenvolvimento desses construtos, separadamente, com pacientes, caso se perceba a importância de trabalhar um ou dois deles com mais profundidade. Ademais, o público em geral pode recorrer a essas práticas como forma de manter a saúde mental e o bem-estar durante o isolamento social. Uma importante vantagem das práticas propostas pela Psicologia Positiva é o baixo custo, além da facilidade de implementação e do relativo desconhecimento de efeitos adversos (Sin \& Lyubomirsky, 2009). 
Outras técnicas, como práticas de gratidão, generosidade, altruísmo e apoio social (não tratados neste artigo por limitação de espaço), podem contribuir substancialmente para o aumento do bem-estar (Layous, Nelson, \& Lyubomirsky, 2013; Sin \& Lyubomirsky, 2009). Ressalta-se que a obtenção de resultados satisfatórios requer mudança de hábitos e estilo de vida, de forma a aprimorar uma expectativa mais positiva sobre si mesmo, sobre a vida e sobre como lidar com as pessoas e situações. Ademais, as práticas mencionadas previamente neste artigo requerem persistência e a criação de uma rotina que as incorpore no dia a dia. Nesse sentido, o suporte social de pessoas importantes, que pode ocorrer de modo online na situação de pandemia, juntamente com o desenvolvimento de atitudes e crenças favoráveis sobre aspectos positivos e aprendizagens decorrentes do isolamento social, constituem importantes fatores para obtenção de melhoria da saúde mental (Birtwell, Williams, van Marwijk, Armitage, \& Sheffield, 2019). Em tempos de isolamento social, o uso de comunicação digital, principalmente com os recursos de imagem e som, constitui um importante meio para manter as relações sociais com familiares, amigos e colegas de trabalho. Ademais, evitar exposição a notícias na televisão e redes sociais pode reduzir o estresse e a ansiedade (van den Bulck \& Custers, 2009) nesse contexto da pandemia.

Apesar dos resultados animadores obtidos com intervenções positivas em contextos diversos (Rashid \& Seligman, 2018), uma importante limitação desta proposta deve-se à falta de resultados empíricos sobre o desenvolvimento de construtos investigados pela Psicologia Positiva na situação da pandemia de COVID-19. Por essa razão, considera-se essencial que estudos de intervenção - controlando diferentes populações e faixas etárias; tipos de práticas, com diferentes combinações; frequência e intensidade do desenvolvimento das práticas - sejam conduzidos durante a pandemia para que se possam obter resultados específicos para o contexto brasileiro. Ressalta-se que as práticas aqui apresentadas podem ser implementadas de forma autônoma por pessoas com ou sem treinamento psicológico.

Este artigo não objetiva apresentar amplamente todas as áreas da Psicologia Positiva (Lopez \& Snyder, 2009; Rashid \& Seligman, 2018), sendo que a seleção desses construtos é arbitrária, tanto por não haver uma indicação de ordem de importância quanto por eles se apresentarem bastante interrelacionados na literatura. A apresentação separada desses construtos deve-se a fins didáticos. Sugere-se que a implementação de estratégias para lidar com dificuldades, aumentar o bem-estar e reduzir sintomas de ansiedade, depressão ou estresse, seja feita de forma harmônica e respeitando o tempo disponível e as necessidades de cada um.

Por fim, apesar dos muitos efeitos adversos causados pelo isolamento social, sugere-se atenção aos aspectos positivos que a situação promove: (a) a possibilidade de passar mais tempo consigo mesmo, o que permite refletir sobre novas estratégias para a autorrealização, como a busca de novos hobbies ou atividades que tragam satisfação ou desafios; (b) a oportunidade de estar mais junto dos filhos e poder ter mais tempo para escutar e compreender as suas emoções; e (c) a visualização criativa do futuro, permitindo imaginar novos tempos em que a pandemia já faz parte do passado. A adoção dessa perspectiva pode promover saúde mental e bem-estar em muitas pessoas.

\section{Contribuição}

C. ZANON e L. L. DELLAZZANA-ZANON contribuíram na concepção, escrita e revisão do artigo. S. M. WECHSLER e R. R. FABRETTI contribuíram na redação de partes do artigo e revisão final. K. N. ROCHA contribuiu na redação de partes do artigo. 


\section{Referências}

Allen, A. B., \& Leary, M. R. (2010). Self-compassion, stress, and coping. Social and Personality Psychology Compass, 4(2), 107-118. http://dx.doi.org/10.1111/j.1751-9004.2009.00246.x

Almeida, L. S., \& Wechsler, S. M. (2015). Excelência profissional: a convergência necessária de variáveis psicológicas. Estudos de Psicologia (Campinas), 32(4), 767-775. http://dx.doi.org/10.1590/0103-166X2015000400019

Bai, Y., Lin, C. C., Lin, C. Y., Chen, J. Y., Chue, C. M., \& Chou, P. (2004). Survey of stress reactions among health care workers involved with the SARS outbreak. Psychiatric Services, 55(9), 1055-1057. http://dx.doi.org/10.1176/appi.ps.55.9.1055

Bajaj, B., Gupta, R., \& Pande, N. (2016). Self-esteem mediates the relationship between mindfulness and wellbeing. Personality and Individual Differences, 94, 96-100. http://dx.doi.org/10.1016/j.paid.2016.01.020

Barnard, L. K., \& Curry, J. F. (2011). Self-compassion: conceptualizations, correlates, and interventions. Review of General Psychology, 15(4), 289-303. http://dx.doi.org/10.1037/a0025754

Beck, J. S. (2011). Cognitive behavior therapy: basics and beyond (2nd ed.). New York: Guilford Press.

Bergin, A. J., \& Pakenham, K. I. (2016). The stress-buffering role of mindfulness in the relationship between perceived stress and psychological adjustment. Mindfulness, 7(4), 928-939. http://dx.doi.org/10.1007/s12671-016-0532-x

Birtwell, K., Williams, K., van Marwijk, H., Armitage, C. J., \& Sheffield, D. (2019). An exploration of formal and informal mindfulness practice and associations with wellbeing. Mindfulness, 10(1), 89-99. http://dx.doi.org/10.1007/s12671-018-0951-y

Bishop, S. R., Lau, M. A., Shapiro, S., Carlson, L., Anderson, N. D., Carmody, J., ... Devins, G. (2004). Mindfulness: a proposed operational definition. Clinical Psychology: Science and Practice, 11, 230-241. http://dx.doi.org/10.1093/clipsy.bph077

Bonanno, G. A. (2004). Loss, trauma, and human resilience: have we underestimated the human capacity to thrive after extremely aversive events? American Psychologist, 59(1), 20-28. http://dx.doi.org/10.1037/0003-066X.59.1.20

Brooks, S. K., Webster, R. K., Smith, L. E., Woodland, L., Wessely, S., Greenberg, N., \& Rubin, G. J. (2020). The psychological impact of quarantine and how to reduce it: rapid review of the evidence. The Lancet, 395(10227), 912-920. http:// dx.doi.org/10.1016/S0140-6736(20)30460-8

Campos, H. M. (2016). O papel da criatividade na mediação do relacionamento entre a paixão empreendedora e a prontidão empreendedora. Revista Brasileira de Gestão de Negócios, 18(61), 457-472. http://dx.doi.org/10.7819/ rbgn.v18i61.3010

Carpena, M. X., Tavares, P. S., \& Menezes, C. B. (2019). The effect of a six-week focused meditation training on depression and anxiety symptoms in Brazilian university students with 6 and 12 months of follow-up. Journal of Affective Disorders, 246, 401-407. http://dx.doi.org/10.1016/j.jad.2018.12.126

Christie, A. M., Atkins, P. W. B., \& Donald, J. N. (2017). The meaning and doing of mindfulness: the role of values in the link between mindfulness and well-being. Mindfulness, 8(2), 368-378. http://dx.doi.org/10.1007/s12671-016-0606-9

Csikszentmihalyi, M. (2014). The systems model of creativity: the collected works of Mihaly Csikszentmihalyi. Dordrecht: Springer.

Desclaux, A., Badji, D., Ndione, A. G., \& Sow, K. (2017). Accepted monitoring or endured quarantine? Ebola contacts' perceptions in Senegal. Social Science and Medicine, 178, 38-45. http://dx.doi.org/10.1016/j.socscimed.2017.02.009

Diener, E. (2000). Subjective well-being: the science of happiness and a proposal for a national index. American Psychologist, 55(1), 34-43. http://dx.doi.org/10.1037/0003-066X.55.1.34

Diener, E., \& Chan, M. Y. (2011) Happy people live longer: subjective well-being contributes to health and longevity. Applied Psychology: Health and Well-Being, 3(1), 1-43. http://dx.doi.org/10.1111/j.1758-0854.2010.01045.x

Diener, E., Heintzelman, S. J., Kushlev, K., Tay, L., Wirtz, D., Lutes, L. D., \& Oishi, S. (2016). Findings all psychologists should know from the new science on subjective well-being. Canadian Psychology, 58(2), 87-104. http://dx.doi.org/10.1037/cap0000063

Fleith, D. S. (2019). The role of creativity in graduate education according to students and professors. Estudos de Psicologia (Campinas), 36, e180045. http://dx.doi.org/10.1590/1982-0275201936e180045

Gallagher, M. W., Lopez, S. J., \& Pressman, S. D. (2013). Optimism is universal: exploring presence and benefits of optimism in a representative sample of the world. Journal of Personality, 81(5), 429-440. http://dx.doi.org/10.1111/jopy.12026

Gillam, T. (2013). Creativity and mental health care. Mental Health Practice, 16(9), 24-30. http://dx.doi.org/10.7748/ mhp2013.06.16.9.24.e807

Glaveanu, V. P. (2014). The Palgrave handbook of creativity and culture research. London: Palgrave Macmillan.

Gondim, S. M., Loiola, E. M., Franciane A. D., Silvia, C. C, Rovira, D. P., Rodrígues, M., ... Mourão, L. (2015). Creativity and innovation as defined by workers. Revista Eletrônica de Administração, 21(3), 549-575. http://dx.doi.org/10.1590/1413-2311.0162015.55629 
González-Rey, F. L. (2018). Vygotsky's "The Psychology of Art": a foundational and still unexplored text. Estudos de Psicologia (Campinas), 35(4), 339-350. http://dx.doi.org/10.1590/1982-02752018000400002

Good, D. J., Lyddy, C. J., Glomb, T. M., Bono, J. E., Brown, K. W., Duffy, M. K., ... Lazar, S. W. (2016). Contemplating mindfulness at work: an integrative review. Journal of Management, 42(1), 114-142. http://dx.doi.org/10.1177/0149206315617003

Hawryluck, L., Gold, W. L., Robinson, S., Pogorski, S., Galea, S., \& Styra, R. (2004). SARS control and psychological effects of quarantine, Toronto, Canada. Emerging Infectious Diseases, 10(7), 1206-1212. http://dx.doi.org/10.3201/ eid1007.030703

Huang, C., Wang, Y., Li, X., Ren, L., Zhao, J., Hu, Y., ... Cao, B. (2020). Clinical features of patients infected with 2019 novel coronavirus in Wuhan, China. The Lancet, 395(10223), 497-506. http://dx.doi.org/10.1016/S0140-6736(20)30183-5

Hutz, C. S., Midgett, A., Pacico, J. C., Bastianello, M. R., \&Zanon, C. (2014). The relationship of hope, optimism, self-esteem, subjective well-being, and personality in Brazilians and Americans. Psychology, 5(6), 514-522. http://dx.doi.org/10.4236/psych.2014.56061

Ivtzan, I., Lomas, T., Hefferon, K., \& Worth, P. (2016). Second wave positive psychology embracing the dark side of life. Abingdon: Routledge.

Jeong, H., Yim, H. W., Song, Y. J., Ki, M., Min, J. A., Cho, J., \& Chae, J. H. (2016). Mental health status of people isolated due to Middle East Respiratory Syndrome. Epidemiology and Health, 38, e2016048. http://dx.doi.org/10.4178/epih. e2016048

Johal, S. S. (2009). Psychosocial impacts of quarantine during disease outbreaks and interventions that may help to relieve strain. The New Zealand Medical Journal, 122(1296), 47-52. Retrieved from https://www.ncbi.nlm.nih.gov/ pubmed/19652680

Kalisch, R., Baker, D. G., Basten, U., Boks, M. P., Bonanno, G. A., Brummelman, E., ... Kleim, B. (2017). The resilience framework as a strategy to combat stress-related disorders. Nature Human Behaviour, 1(11), 784-790. http://dx. doi.org/10.1038/s41562017-0200-8

Kaufman, J. C. \& Sternberg, R. J. (2010). The Cambridge handbook of creativity. New York: Cambridge University Press.

Layous, K., Nelson, S. K., \& Lyubomirsky, S. (2013). What is the optimal way to deliver a positive activity intervention? The case of writing about one's best possible selves. Journal of Happiness Studies, 14(2), 635-654. http://dx.doi.org/10.1007/s10902-012-9346-2

Leary, M. R., Tate, E. B., Adams, C. E., Allen, A. B., \& Hancock, J. (2007). Self-compassion and reactions to unpleasant self-relevant events: the implications of treating oneself kindly. Journal of Personality and Social Psychology, 92(5), 887-904. http://dx.doi. org/10.1037/0022-3514.92.5.887

Li, Q., Guan, X., Wu, P., Wang, X., Zhou, L., Tong, Y., ... Feng, Z. (2020). Early transmission dynamics in Wuhan, China, of novel coronavirus infected pneumonia. New England Journal of Medicine, 382(13), 1199-1207. http://dx.doi. org/10.1056/NEJMoa2001316

Lopez, S. J., \& Snyder, C. R. (2009). Oxford handbook of positive psychology (2nd ed.). New York: Oxford University Press.

Luthar, S. S., Cicchetti, D., \& Becker, B. (2000). The construct of resilience: a critical evaluation and guidelines for future work. Child Development, 71, 543-562. http://dx.doi.org/10.1111/1467-8624.00164

Luthar, S. S., Lyman, E. L., \& Crossman, E. J. (2014). Resilience and positive psychology. In M. Lewis \& K. Rudolph (Eds.), Handbook of developmental psychopathology (pp.125-140). New York: Springer.

Lyubomirsky, S. (2007). The how of happiness: a scientific approach to getting the life you want. New York: Penguin Press.

Lyubomirsky, S. (2019). The road to happiness is paved with a little gold, a lot of reporters, many emails, thousands of frequent flyer miles, and 604 hours of writing. Perspectives on Psychological Science, 14(1), 26-28. http://dx.doi. org/10.1177/1745691618808516

Lyubomirsky, S., \& Layous, K. (2013). How do simple positive activities increase well-being? Current Directions in Psychological Science, 22(1), 57-62. http://dx.doi.org/10.1177/0963721412469809

Lyubomirsky, S., Sheldon, K. M., \& Schkade, D. (2005). Pursuing happiness: the architecture of sustainable change. Review of General Psychology, 9(2), 111-131. http://dx.doi.org/10.1037/1089-2680.9.2.111

Ma, L., Zhang, Y., \& Cui, Z. (2019). Mindfulness-based Interventions for prevention of depressive symptoms in university students: a meta-analytic Review. Mindfulness, 10(11), 2209-2224. http://dx.doi.org/10.1007/s12671-019-01192-w

Masten, A. S. (2001). Ordinary magic: resilience processes in development. American Psychologist, 56(3), 227-238. http:// dx.doi.org/10.1037/0003-066X.56.3.227

Masten, A. S., \& Reed, M. J. (2002). Resilience in development. In C. R. Snyder \& S. J. Lopez (Eds.), Handbook of positive psychology (pp.74-88). London: Oxford University Press.

Menezes, C. B., Dell'Aglio, D. D., \& Bizarro, L. (2011). Meditação, bem-estar e a ciência psicológica: revisão de estudos empíricos. Interação em Psicologia, 15(2). http://dx.doi.org/10.5380/psi.v15i2.20249 
Mihashi, M., Otsubo, Y., Yinjuan, X., Nagatomi, K., Hoshiko, M., \& Ishitake, T. (2009). Predictive factors of psychological disorder development during recovery following SARS outbreak. Health Psychology, 28(1), 91-100. http://dx.doi. org/10.1037/a0013674

Morais, M. F., \& Almeida, L. (2019). "I would be more creative if..." : are there perceived barriers to college students' creative expression according to gender? Estudos de Psicologia (Campinas), 36, e180011. http://dx. doi.org/10.1590/1982-0275201936e180011

Morina, N., Stam, K., Pollet, T. V., \& Priebe, S. (2018). Prevalence of depression and posttraumatic stress disorder in adult civilian survivors of war who stay in war-afflicted regions: a systematic review and meta-analysis of epidemiological studies. Journal of Affective Disorders, 239, 328-338. http://dx.doi.org/10.1016/j.jad.2018.07.027

Mundim, M. C., Wechsler, S. M., \& Morais, M. F. (2020). Environmental and psychological factors that influence the creative excellence of Brazilian and Portuguese women. The Journal of Creative Behavior [Ahead of print]. http:// dx.doi.org/10.1002/jocb.43

Nakano, T. C., Machado, W. L., \& Abreu, I. C. C. (2019). Relações entre estilos de pensar e criar, bem-estar, saúde percebida e estresse na terceira idade. Psico-USF, 24(3), 555-568. http://dx.doi.org/10.1590/1413-82712019240312

Nakano, T. C., \& Wechsler, S. M. (2018). Creativity and innovation: skills for the 21st Century. Estudos de Psicologia (Campinas), 35(3), 237-246. http://dx.doi.org/10.1590/1982-02752018000300002

Neff, K. D. (2003a). The development and validation of a scale to measure self-compassion. Self and Identity, 2, 223-250. http://dx.doi.org/10.1080/15298860309027

Neff, K. D. (2003b). Self-compassion: an alternative conceptualization of a healthy attitude toward oneself. Self and Identity, 2, 85-102. Retrieved from https://psycnet.apa.org/doi/10.1080/15298860309032

Neff, K. D., \& Germer, C. (2017). Self-compassion and psychological wellbeing. In J. Doty (Ed.), Oxford handbook of compassion science (pp.371-386). New York: Oxford University Press.

Oliveira, K. S., Nakano, T. C., \& Wechsler, S. (2016). Criatividade e saúde mental: uma revisão da produção cientifica na última década. Temas em Psicologia, 24(4), 1493-1506. http://dx.doi.org/10.9788/TP2016.4-16

Osborn, A. F. (1963). Applied imagination. New York: Scribner.

Parnes, S. J. (1967). The literature of creativity (part II). The Journal of Creative Behavior, 1(2), 191-240. http://dx.doi. org/10.1002/j.2162-6057.1967.tb00027.x

Pelechano, V., González-Leandro, P., García, L., \& Morán, C. (2013). Is it possible to be too happy? Happiness, personality, and psychopathology. International Journal of Clinical and Health Psychology, 13(1), 18-24. http://dx. doi.org/10.1016/S16972600(13)70003-6

Peterson, C., Ruch, W., Beermann, U., Park, N., \& Seligman, M. E. P. (2007). Strengths of character, orientations to happiness, and life satisfaction. The Journal of Positive Psychology, 2(3), 149-156. http://dx.doi.org/10.1080/17439760701228938

Peterson, C., \& Seligman, M. E. P. (2004). Character strengths and virtues: a handbook and classification. Washington: American Psychological Association.

Peterson, C., \& Steen, T. A. (2002). Optimistic explanatory style. In C. R. Snyder \& S. J. Lopez (Eds.), Handbook of positive psychology (pp.244-256). Oxford: Oxford University Press.

Qiu, J., Shen, B., Zhao, M., Wang, Z., Xie, B., \& Xu, Y. (2020). A nationwide survey of psychological distress among Chinese people in the COVID-19 epidemic: implications and policy recommendations. General Psychiatry, 33(2), e100213. http://dx.doi.org/10.1136/gpsych-2020-100213

Rashid, T., \& Seligman, M. P. (2018). Positive psychotherapy: clinical manual. New York: Oxford University Press.

Reynolds, D. L., Garay, J. R., Deamond, S. L., Moran, M. K., Gold, W., \& Styra, R. (2008). Understanding, compliance and psychological impact of the SARS quarantine experience. Epidemiology \& Infection, 136(7), 997-1007. http:// dx.doi.org/10.1017/S0950268807009156

Robinson, C., \& Snipes, K. (2009). Hope, optimism and self-efficacy: a system of competence and control enhancing African American college student's academic well-being. Multiple Linear Regression Viewpoints, 35, 16-26.

Rosin, A. B., Zanon, C., \& Teixeira, M. A. P. (2014). Bem-estar subjetivo, personalidade e vivências acadêmicas em estudantes universitários. Interação em Psicologia, 1(18), 1-12. http://dx. doi.org/10.5380/psi.v18i1.27634

Runco, M. A., \& Pritzker, S. R. (2020). Encyclopedia of creativity (3rd ed). New York: Academic Press.

Santos, M. C., Gibim, Q. G. M. T., \& Wechsler, S. M. (2020). Relação entre criatividade e otimismo: percepção de adultos. Revista Iberoamericana de Criatividade e Inovação, 1(1), 41-50. Recuperado de https://recriai.emnuvens.com.br/revista/ article/download/23/8

Seligman, M. E. P., \& Csikszentmihalyi, M. (2000). Positive psychology: an introduction. American Psychologist, 55(1), 5-14. http://dx.doi.org/10.1037/0003-066X.55.1.5 
Sheldon, K. M., \& Lyubomirsky, S. (2019). Revisiting the sustainable happiness model and pie chart: can happiness be successfully pursued? The Journal of Positive Psychology, 1-10. http://dx.doi.org/10.1080/17439760.2019.1689421

Shu, K., Wang, S., \& Liu, H. (2018). Beyond news contents: the role of social context for fake news detection. ArXiv. Version 2. Retrieved from http://arxiv.org/abs/1712.07709

Sin, N. L., \& Lyubomirsky, S. (2009). Enhancing well-being and alleviating depressive symptoms with positive psychology interventions: a practice-friendly meta-analysis. Journal of Clinical Psychology, 65(5), 467-487. http://dx.doi.org/10.1002/ jclp.20593

Smirnova, M., \& Parks, A. C. (2018). Positive psychology interventions: clinical applications. In D. S. Dunn (Ed.), Positive psychology: established and emerging issues (pp.276-297). Abingdon: Routledge.

Snyder, C. R., \& Lopez, S. A. (2008). Psicologia positiva: uma abordagem cientifica e prática das qualidades humanas. Porto Alegre: Artmed.

Souza, L. K., \& Hutz, C. S. (2016). Self-compassion in relation to self-esteem, self-efficacy and demographical aspects. Paidéia, 26(64), 181-188. http://dx.doi.org/10.1590/1982-43272664201604

Souza, L. K., Policarpo, D., \& Hutz, C. S. (2020). Self-compassion and symptoms of stress, anxiety, and depression [Ahead of print]. Trends in Psychology. http://dx.doi.org/10.1007/s43076-020-00018-2

Sprang, G., \& Silman, M. (2013). Posttraumatic stress disorder in parents and youth after health-related disasters. Disaster Medicine and Public Health Preparedness, 7(1), 105-110. http://dx.doi.org/10.1017/dmp.2013.22

Tomlinson, E. R., Yousaf, O., Vittersø, A. D., \& Jones, L. (2018). Dispositional mindfulness and psychological health: a systematic review. Mindfulness, 9(1), 23-43. http://dx.doi.org/10.1007/s12671-017-0762-6

van den Bulck, J., \& Custers, K. (2009). Television exposure is related to fear of avian flu, an Ecological Study across 23 member states of the European Union. European Journal of Public Health, 19(4), 370-374. http://dx.doi.org/10.1093/ eurpub/ckp061

Wang, C., Pan, R., Wan, X., Tan, Y., Xu, L., McIntyre, R. S., .. Ho, C. (2020a). A longitudinal study on the mental health of general population during the COVID-19 epidemic in China. Brain, Behavior, and Immunity. http://dx.doi. org/10.1016/j.bbi.2020.04.028

Wang, D., Hu, B., Hu, C., Zhu, F., Liu, X., Zhang, J., ... Peng, Z. (2020b). Clinical characteristics of 138 hospitalized patients with 2019 novel coronavirus: infected pneumonia in Wuhan, China. JAMA, 323(11), 1061. http://dx.doi. org/10.1001/jama.2020.1585

Watson, D., \& Clark, L. A. (1994). The PANAS-X: manual for the positive and negative affect schedule-Expanded form. lowa City: Department of Psychological \& Brain Sciences Publications. http://dx.doi.org/10.17077/48vt-m4t2

Wechsler, S. M. (2008). Criatividade: descobrindo e encorajando. Campinas: Laser Graphics

World Health Organization. (2020). WHO Director-General's opening remarks at the media briefing on COVID-19-11 March 2020. Geneva: Author. Retrieved from https://www.who.int/dg/speeches/detail/who-director-general-s-openingremarks-at-the-media-briefing-on-covid-19---11-march-2020

Wu, F., Zhao, S., Yu, B., Chen, Y. M., Wang, W., Song, Z. G., ... Zhang, Y. Z. (2020). A new coronavirus associated with human respiratory disease in China. Nature, 579(7798), 265-269. http://dx.doi.org/10.1038/s41586-020-2008-3

Zanon, C. (2017). Felicidade e indicadores de psicopatologia em brasileiros. Avaliação Psicológica, 16(4), 452-457. http:// dx.doi.org/10.15689/ap.2017.1604.12857

Zanon, C., Dellazzana-Zanon, L. L., \& Hutz, C. S. (2014). Afetos positivos e negativos: definições, avaliações e suas implicações para intervenções. In C. S. Hutz (Org.), Avaliação em psicologia positiva (pp.49-62). Porto Alegre: Artmed.

Zanon, C., Hutz, C. S., Reppold, C. T., \& Zenger, M. (2016). Are happier people less vulnerable to rumination, anxiety, and post-traumatic stress? evidence from a large scale disaster. Psicologia: Reflexão e Crítica, 29(1), 1-7. http://dx.doi. org/10.1186/s41155-016-0038-4

Zimmaro, L. A., Salmon, P., Naidu, H., Rowe, J., Phillips, K., Rebholz, W. N., ... Sephton, S. E. (2016). Association of dispositional mindfulness with stress, cortisol, and well-being among university undergraduate students. Mindfulness, 7(4), 874-885. http://dx.doi.org/10.1007/s12671-016-0526-8

Recebido: abril 13, 2020

Versão final: maio 4, 2020

Aprovado: maio 7, 2020 